\title{
STABLE MATCHINGS IN THE MARRIAGE MODEL WITH INDIFFERENCES *
}

\author{
by \\ Noelia Juarez $^{\dagger},{ }^{\dagger}$ Jorge Oviedo $^{\dagger}$
}

2019

ABSTRACT: For the marriage model with indifferences, we define an equivalence relation over the stable matching set. We identify a sufficient condition, the closing property, under which we can extend results of the classical model (without indifferences) to the equivalence classes of the stable matching set. This condition allows us to extend the lattice structure over classes of equivalences and the rural hospital theorem.

\section{Introduction}

The marriage model describes a matching problem in which agents are divided into two disjoint subsets: the set of men and the set of women. The objective of this model is to assign a woman to a man, allowing the possibility for men and/or women to stay single or without a partner. In this paper, we study the marriage model when agents in both sides of the market may be indifferent to agents of the other side.

Most papers dealing with matching models assume that agents are not indifferent to the agents on the other side of the market. Many results for the matching model when preferences are strict cannot be extended to the

* Our work is partially supported by Universidad Nacional de San Luis through grant 31012, and by the Consejo Nacional de Investigaciones Científicas y Técnicas (CONICET), through grant PIP 112-201501-00464.

${ }^{\dagger}$ Instituto de Matemática Aplicada San Luis, IMASL. Universidad Nacional de San Luis and CONICET. Ejército de los Andes 950. D5700HHW San Luis. República Argentina. E-mails: nmjuarez@unsl.edu.ar and joviedo@unsl.edu.ar 
matching model when agents have preferences with indifferences. (See Roth and Sotomayor 1990).

For convenience we use the marriage terminology, but we have in mind other kinds of matchings. Some examples include matching professors to researchassistants: let's suppose that a group of professors and research assistants live in different cities, the preferences of professors (research assistants) over research assistants (professors) depend on the distance between both groups. Then professors are indifferent to all research assistants who live in the same city. These research assistants are strictly preferred to research assistants who live in more distant cities. Other example is matching doctors to nurses, when a nurse is acceptable to a doctor if and only if she has certain skills, and a doctor is acceptable to a nurse if he is not requesting "hard" tasks; or matching pilots to copilots, managers to support staff, and so on. This special case of bilateral matching problem, where each man evaluates each woman as acceptable or unacceptable: acceptable partners are indifferent to each other. It is known in literature as dichotomous preferences (See Bogomolnaia and Moulin 2004).

In matching models, stability is considered the main property to be satisfied by any matching. Unlike the marriage model with strict preferences, where there is a unique concept of stability satisfied by pairs of agents, in the marriage model with indifferences there are several concepts of stability. A matching is stable ${ }^{1}$ if each agent is matched to an acceptable partner, and there is no man-woman pair such that they are not matched together and strictly prefer each other to their current partners. Irving (1994) formulates two other possible definitions of stability for the marriage model with indifferences. A matching is strongly stable if each agent is matched to an acceptable partner, and there is no man-woman pair such that they are not matched together and one of them strictly prefers the other one to their current partner, the other weakly prefers the other one to their current partner. A matching is super stable if each agent is matched to an acceptable partner, and there is no man-woman pair such that they are not matched together and weakly prefer each other to their current partners.

The existence of stable matchings in the marriage model with indifferences is guaranteed: by breaking ties arbitrarily, we obtain a strict marriage model. A stable matching in this strict model is a stable matching in the original

\footnotetext{
${ }^{1}$ Irving (1994) refers to stable matchings as weakly stable matchings. In this paper, we use the term stable matching.
} 
marriage model with indifferences. On the other hand, strongly stable matchings and super stable matchings could not exist. Irving (1994) presents algorithms for determining whether a strongly stable matching and/or a super stable matching exist. In each case, if such a matching exists, the appropriate algorithm constructs one. In this paper, we focus on stable matchings.

Knuth (1976), who attributed the result to Conway, establishes the lattice structure of the stable matching set for the marriage model with strict preferences. Manlove (2002) shows that the strongly stable matching set has a lattice structure when it is partitioned by a suitable equivalence relation. Spieker (1985) proved that the set of super stable matchings forms a distributive lattice. However, in the case of indifference, this structure is absent for stable matchings. We give a sufficient condition, the closing property, under which we prove the lattice structure over equivalence classes of the stable matching set in the model with indifferences. This result is obtained by defining an equivalent relation on the set of matchings.

We also prove that the set of strongly stable matchings and the set of super stable matchings always satisfy the closing property. If the strongly stable matching set is not empty, we prove that the lattice over equivalence classes of strong matchings (Manlove 2002) is a sublattice of the lattice over equivalence classes of stable matchings.

Given $\mu$ and $\mu^{\prime}$ two matchings, the closing property requires that the indifferent agents between $\mu$ and $\mu^{\prime}$ match each other. The closing property has the weakness of being defined on the set of matchings and not on the preferences of the agents. Other authors also use notions based on matchings, to study matching models with indifferences. For instance, Erdil and Ergin $(2008,2017)$ study the notion of cycles and chains on matchings to calculate optimal stable matchings. Sotomayor (2011) use Pareto stability concept to study matching markets with indifferences. Despite this weakness, we show special classes of preferences, under which the closing property holds. The strict preferences satisfy the closing property. We show that the dichotomous preferences also satisfy the closing property. In addition, matching models where one group of agents has dichotomous preferences and another group has strict preferences, satisfy the closing property.

In the hospital resident model with strict preferences, the rural hospital theorem (Roth 1984, 1986) is well known. However, when we allow indifferences in preferences, the rural hospital theorem is not valid. We generalize the rural hospital theorem over all stable matchings, under the closing property. 
The rural hospital theorem states that if one hospital has a vacancy in some stable matching, then the set of residents assigned to it is the same (in particular, we have the same number of vacancies in any stable matching). In the context of the marriage model, the theorem states that the set of agents remaining single is the same for all stable matchings (McVitie and Wilson 1970). The rural hospital theorem is of great importance, since it produces dissatisfaction in stable allocation mechanisms.

Irving, Manlove and Scott (2000) prove that if the set of super stable matchings is not empty, then the rural hospital theorem is valid over all stable matchings. If the set of strongly stable matchings is not empty, since the strongly matching set satisfies the closing property, the rural hospital theorem is valid over all strongly stable matchings.

This paper is organized as follows. In Section 2, we formally describe the model and present some preliminaries, we study matching equivalence classes. In Section 3, we define the closing property and we show the lattice structure over equivalence classes of stable matchings. In Section 4, we extend the rural hospital theorem to the marriage model with indifferences. Finally, we present some concluding comments.

\section{The model}

In the marriage model with indifferences there are two finite sets of agents, $M=\left\{m_{1}, \ldots, m_{n}\right\}$ of men and the sets $W=\left\{w_{1}, \ldots, w_{p}\right\}$ of women. We sometimes denote a generic agent by $i$ and a generic man and a generic woman by $m$ and $w$, respectively. Each agent $i \in M \cup W$, has a complete and transitive preference ordering $R_{i}$ (weak order) over the agents on the other side of the market and the prospect of being alone. We denote by $R=\left(R_{i}\right)_{i \in M \cup W}$ a preference profile of weak orders. We denote by $P_{i}$ and $I_{i}$ the antisymmetric and symmetric parts of the binary relation $R_{i}$. Then $P_{i}$ is antisymmetric, transitive and irreflexive (strict preference relation), and $I_{i}$ is reflexive, symmetric and transitive (indifference preference relation).

We assume that $R$ satisfies no indifference to the single set. This means that any agent is not indifferent to either remaining single or being assigned to another agent of the other side of the market. This assumption is commonly used in the literature; see Erdil and Ergin $(2008,2017)$.

For the man $m$ for instance, the preference $R_{m}$ where $w_{1} P_{m} w_{2}, w_{2} P_{m} w_{3}$, 
$w_{2} P_{m} m$, and $w_{3} I_{m} w_{4}$, and by transitivity $w_{1} P_{m} w_{3}$ and $w_{1} P_{m} w_{4}$, will be denoted by

$$
R_{m}: w_{1}, w_{2},\left[w_{3}, w_{4}\right], m .
$$

A specific marriage model with indifference will be denoted by the triple $(M, W, R)$. A woman $w$ is acceptable to a man $m$ if $w P_{m} m$. Analogously, $m$ is acceptable to $w$ if $m P_{w} w$.

Definition 1 A pre-matching $\nu$ is a correspondence $\nu: M \cup W \rightarrow M \cup W$

1. $\mu(m) \neq m$ implies $\mu(m) \in W$.

2. $\mu(w) \neq w$ implies $\mu(w) \in M$.

Definition 2 A matching $\mu$ is a injective function $\mu: M \cup W \rightarrow M \cup W$

1. $\mu(m) \neq m$ implies $\mu(m) \in W$.

2. $\mu(w) \neq w$ implies $\mu(w) \in M$.

3. $\mu(m)=w$ if and only if $\mu(w)=m .^{2}$

Let $\mathcal{M}$ denote the set of all matchings. If $\mu(m)=w$, then man $m$ and woman $w$ are said to be matched to one another. If $\mu(i)=i$, then agent $i$ is said to be single or unmatched. A pre-matching $\nu$ is a matching such that $\mu(m)=w$ if and only if $\mu(w)=m$.

Given a subset of agents $X \subseteq M \cup W$, we extend the binary relationship $R_{i}, I_{i}$ and $P_{i}$ over the set of matchings as follows:

1. $\mu R_{X} \mu^{\prime}$ when $\mu(i) R_{i} \mu^{\prime}(i)$ for all $i \in X$.

2. $\mu I_{X} \mu^{\prime}$, when $\mu(i) I_{i} \mu^{\prime}(i)$ for all $i \in X$.

3. $\mu P_{X} \mu^{\prime}$ when for all $i \in X, \mu(i) R_{i} \mu^{\prime}(i)$ and there exists $i^{\prime} \in X$ such that $\mu\left(i^{\prime}\right) P_{i^{\prime}} \mu^{\prime}\left(i^{\prime}\right)$.

\footnotetext{
${ }^{2}$ Item 3 is equivalent to say that $\mu$ is a homogenous function of order two, i.e., $\mu^{2}(i)=i$, for all $i \in M \cup W$.
} 
When $X=\{i\}$, for simplicity of notation, we write $\mu R_{i} \mu^{\prime}$ instead of $\mu R_{\{i\}} \mu^{\prime}$. We write the negation of $\mu R_{i} \mu^{\prime}$ as $\overline{\mu R_{i} \mu^{\prime}}$.

In the marriage model with strict preferences $R_{M}$ is a partial order over the set of matchings, i.e. it is a reflexive, antisymmetric and transitive binary relation. Nevertheless, in the marriage model with indifferences $R_{M}$ is not a partial order over the set of matchings since it may occur that $\mu \neq \mu^{\prime}, \mu R_{M} \mu^{\prime}$ and $\mu^{\prime} R_{M} \mu$. In this case $R_{M}$ is not an antisymmetric relation. (See example $1)$.

Consider a matching $\mu$ that matches a pair $(m, w)$. If at least one of the individuals $m$ and $w$ weakly prefers remaining single rather than be matched to the other. Such a matching $\mu$ is blocked by this individual. A matching $\mu$ is individually rational if it is not blocked by any individual agent. Formally, $\mu$ is individually rational for all $i \in M \cup W$ we have that $\mu(i) R_{i} i$. Given a preference profile $R$, we denote the set of individually rational matchings by $I R(R)$.

Definition 3 For a given matching $\mu$

- A pair $(m, w)$ is said to form a blocking pair if $m P_{w} \mu(w)$ and $w P_{m} \mu(m)$.

- A pair $(m, w)$ is said to form a strongly blocking pair if $m P_{w} \mu(w)$ and $w R_{m} \mu(m)$ or $m R_{w} \mu(w)$ and $w P_{m} \mu(m)$.

- A unmatched pair $(m, w)$ is said to form a super blocking pair if $m R_{w} \mu(w)$ and $w R_{m} \mu(m)$.

Definition 4 For a given matching $\mu$

- A matching $\mu$ is stable if it is individually rational and if there is not a blocking pair.

- A matching $\mu$ is strongly stable if it is individually rational and there is not a strongly blocking pair.

- A matching $\mu$ is super stable if it is individually rational and there is not a super blocking pair.

Given a preference profile $R$, we denote the set of stable matchings by $S(R)$. We denote the set of strongly stable matchings and super stable matchings by $S S(R)$ and $S S S(R)$ respectively.

It follows from the definition $S S S(R) \subseteq S S(R) \subseteq S(R)$. 


\section{Classes of matchings}

We define the classes of matchings and we will extend these binary relations $R_{i}, I_{i}$ and $P_{i}$ to the classes of matchings. Given two matchings $\mu$ and $\mu^{\prime}$, we define the following equivalence binary operation:

$$
\mu \sim \mu^{\prime} \Longleftrightarrow \mu(i) I_{i} \mu^{\prime}(i) \text { for all } i \in M \cup W \text {. }
$$

Given a matching $\mu$ we denote the equivalence class of $\mu$,

$$
[\mu]=\left\{\mu^{\prime} \in \mathcal{M}: \mu \sim \mu^{\prime}\right\} .
$$

Observe that $\mathcal{M}=\cup_{\mu}[\mu]$ is a partition of $\mathcal{M}$.

Example 1 Let $M=\left\{m_{1}, m_{2}\right\}$ be the set of men and $W=\left\{w_{1}, w_{2}\right\}$ be the set of women. Consider the preference profile $R$ :

$$
\begin{array}{ll}
R_{m_{1}}:\left[w_{1}, w_{2}\right], m_{1} . & R_{w_{1}}:\left[m_{1}, m_{2}\right], w_{1} . \\
R_{m_{2}}:\left[w_{1}, w_{2}\right], m_{2} . & R_{w_{2}}:\left[m_{1}, m_{2}\right], w_{2} .
\end{array}
$$

The set of stable matchings consists of the following two matchings:

$$
\mu_{1}=\left(\begin{array}{cc}
m_{1} & m_{2} \\
w_{1} & w_{2}
\end{array}\right) \text { and } \mu_{2}=\left(\begin{array}{cc}
m_{1} & m_{2} \\
w_{2} & w_{1}
\end{array}\right)
$$

Observe that $\mu_{1} R_{M} \mu_{2}$ and $\mu_{2} R_{M} \mu_{1}$. Nevertheless $\mu \neq \mu^{\prime}$. In this example $\mu_{1} I_{M} \mu_{2}$, and $\mu_{1} I_{W} \mu_{2}$, i.e. $\left[\mu_{1}\right]=\left[\mu_{2}\right]$. Notice that $R_{M}$ is not an antisymmetric relation over the stable matching set, then $R_{M}$ is not a partial order over $S(R)$.

The following Lemma is useful for extending the binary relations to classes of matchings.

Lemma 1 Let $(M, W, R)$ be a marriage model with indifferences. Let $\mu_{1}$, $\mu_{2}, \mu_{3} \in M$ for all $i \in M \cup W$

1. If $\mu_{1} I_{i} \mu_{2}$ and $\mu_{2} P_{i} \mu_{3}$ then $\mu_{1} P_{i} \mu_{3}$.

2. If $\mu_{1} P_{i} \mu_{2}$ and $\mu_{2} I_{i} \mu_{3}$ then $\mu_{1} P_{i} \mu_{3}$.

3. If $\mu_{1} P_{i} \mu_{2}$ then $\mu_{1}^{\prime} P_{i} \mu_{2}^{\prime}$ for all $\mu_{i}^{\prime} \in\left[\mu_{i}\right], i=1,2$. 
4. If $\mu_{1} R_{i} \mu_{2}$ then $\mu_{1}^{\prime} R_{i} \mu_{2}^{\prime}$ for all $\mu_{i}^{\prime} \in\left[\mu_{i}\right], i=1,2$.

See the Appendix, for a proof. Given $X \subseteq M \cup W$, now as consequence of Lemma 1, we can extend $R_{i}, I_{i}$ and $P_{i}$ to classes of matchings, in the following form:

1. $\left[\mu_{1}\right] R_{X}\left[\mu_{2}\right]$ if and only if $\mu_{1} R_{i} \mu_{2}$ for all $i \in X$.

2. $\left[\mu_{1}\right] I_{X}\left[\mu_{2}\right]$ if and only if $\mu_{1} I_{i} \mu_{2}$ for all $i \in X$.

3. $\left[\mu_{1}\right] P_{X}\left[\mu_{2}\right]$ if and only if $\left[\mu_{1}\right] R_{X}\left[\mu_{2}\right]$ and there exists $i^{\prime} \in X$ such that $\mu_{1} P_{i^{\prime}} \mu_{2}$.

For simplicity of notation, we write $\left[\mu_{1}\right] R_{i}\left[\mu_{2}\right]$ instead of $\left[\mu_{1}\right] R_{\{i\}}\left[\mu_{2}\right]$. When $X=M \cup W$ we write $\left[\mu_{1}\right] R\left[\mu_{2}\right]$ instead of $\left[\mu_{1}\right] R_{M \cup W}\left[\mu_{2}\right]$.

Given a preference profile $R$, we denote by $S(R) / \sim=\{[\mu]: \mu \in S(R)\}$ the quotient set.

Now we show that $R_{M}^{3}$ is a partial order over the quotient set $S(R) / \sim$.

Proposition 1 Let $(M, W, R)$ be a marriage model with indifferences. Then $R_{M}$ is a partial order over $S(R) / \sim$, i.e. it is a reflexive, antisymmetric and transitive binary relation.

See the Appendix for a proof.

\section{The structure of stable matchings}

When preferences are strict the set of stable matchings $S(P)$ forms a lattice under the partial order $R_{M}$. This structure is absent when we allow indifferences in preferences, see Example 3. In order to establish the existence of a lattice structure, we need to define the closing property for matchings.

\footnotetext{
${ }^{3}$ By abuse of notation, we continue to write $R_{M}$ for a order over $S(R) / \sim$.
} 


\section{The closing property}

Given $\mu, \mu^{\prime} \in \mathcal{M}$, we define the following sets:

$$
\begin{aligned}
& J_{M}\left(\mu, \mu^{\prime}\right)=\left\{m \in M: \mu(m) \neq \mu^{\prime}(m), \text { and } \mu(m) I_{m} \mu^{\prime}(m)\right\}, \\
& J_{W}\left(\mu, \mu^{\prime}\right)=\left\{w \in M: \mu(w) \neq \mu^{\prime}(w), \text { and } \mu(w) I_{w} \mu^{\prime}(w)\right\} .
\end{aligned}
$$

Definition 5 Given $T \subseteq \mathcal{M}$ we say that $T$ satisfies the closing property if for all $\mu, \mu^{\prime} \in T$

$$
\mu\left(J_{M}\left(\mu, \mu^{\prime}\right)\right)=J_{W}\left(\mu, \mu^{\prime}\right) .
$$

Definition 5 specifies that a subset of matchings satisfies the closing property if given two matchings $\mu$ and $\mu^{\prime}$, the women that are indifferent between $\mu$ and $\mu^{\prime}$ are matched to men who are also indifferent between $\mu$ and $\mu^{\prime}$. It means that the indifferent agents between $\mu$ and $\mu^{\prime}$ are matched to each other.

When $T=S S(R)$ and $T=S S S(R)$, we will show in Proposition 4 and 5 , that $S S(R)$ and $S S S(R)$ always satisfy the closing property. However the stable matching set $S(R)$, sometimes does not satisfy the closing property (See Example 2).

The following Lemma states that we can exchange the role of $M$ by $W$ in Definition 5.

Lemma 2 Given $\mu, \mu^{\prime} \in \mathcal{M}$,

$$
\mu\left(J_{M}\left(\mu, \mu^{\prime}\right)\right)=J_{W}\left(\mu, \mu^{\prime}\right) \text { if and only if } \mu\left(J_{W}\left(\mu, \mu^{\prime}\right)\right)=J_{M}\left(\mu, \mu^{\prime}\right) \text {. }
$$

Proof $\Longrightarrow)$ Suppose that $\mu\left(J_{M}\left(\mu, \mu^{\prime}\right)\right)=J_{W}\left(\mu, \mu^{\prime}\right)$. I.e.

$$
\mu\left(J_{M}\left(\mu, \mu^{\prime}\right)\right) \subseteq J_{W}\left(\mu, \mu^{\prime}\right) \text { and } J_{W}\left(\mu, \mu^{\prime}\right) \subseteq \mu\left(J_{M}\left(\mu, \mu^{\prime}\right)\right) .
$$

We apply $\mu$,

$$
\mu^{2}\left(J_{M}\left(\mu, \mu^{\prime}\right)\right) \subseteq \mu\left(J_{W}\left(\mu, \mu^{\prime}\right)\right) \text { and } \mu\left(J_{W}\left(\mu, \mu^{\prime}\right)\right) \subseteq \mu^{2}\left(J_{M}\left(\mu, \mu^{\prime}\right)\right) .
$$

Since $\mu$ and $\mu^{\prime}$ are homogenous functions of order two we have that

$$
J_{M}\left(\mu, \mu^{\prime}\right) \subseteq \mu\left(J_{W}\left(\mu, \mu^{\prime}\right)\right) \text { and } \mu\left(J_{W}\left(\mu, \mu^{\prime}\right)\right) \subseteq J_{M}\left(\mu, \mu^{\prime}\right) .
$$


Hence $\mu\left(J_{W}\left(\mu, \mu^{\prime}\right)\right)=J_{M}\left(\mu, \mu^{\prime}\right)$.

$\Longleftarrow)$ The proof is similar to the previous one.

The following example shows that $S(R)$ does not satisfy the closing property.

Example 2 Let $M=\left\{m_{1}, m_{2}\right\}$ be the set of men and $W=\left\{w_{1}, w_{2}\right\}$ be the set of women. Consider the preference profile $R$ :

$$
\begin{array}{ll}
R_{m_{1}}: w_{1}, w_{2}, m_{1} . & R_{w_{1}}: m_{2}, m_{1}, w_{1} \\
R_{m_{2}}:\left[w_{1}, w_{2}\right], m_{2} . & R_{w_{2}}: m_{1}, m_{2}, w_{2}
\end{array}
$$

The set of stable matchings consists of the following two matchings:

$$
\mu_{1}=\left(\begin{array}{ll}
m_{1} & m_{2} \\
w_{1} & w_{2}
\end{array}\right) \text { and } \mu_{2}=\left(\begin{array}{ll}
m_{1} & m_{2} \\
w_{2} & w_{1}
\end{array}\right) .
$$

Observe that

$$
J_{M}\left(\mu_{1}, \mu_{2}\right)=\left\{m_{2}\right\} \text { and } J_{W}\left(\mu_{1}, \mu_{2}\right)=\emptyset .
$$

Moreover, $\mu_{1}\left(J_{M}\left(\mu_{1}, \mu_{2}\right)\right)=\left\{w_{2}\right\}$. So $\mu_{1}\left(J_{M}\left(\mu_{1}, \mu_{2}\right)\right) \neq J_{W}\left(\mu_{1}, \mu_{2}\right)$. We have that $S(R)$ does not satisfy the closing property. Since $S S(R)=\left\{\mu_{2}\right\}$, we have that $S S(R)$ satisfies the closing property.

Proposition 2 Let $(M, W, P)$ be a marriage model with strict preferences, then $S(P)$ satisfies the closing property.

Proof Let $\mu, \mu^{\prime} \in S(P)$. We assume for any agent $\mu(i) I_{i} \mu^{\prime}(i)$. Since the preferences are strict, $\mu(i)=\mu^{\prime}(i)$. Hence $J_{M}\left(\mu, \mu^{\prime}\right)=J_{W}\left(\mu, \mu^{\prime}\right)=\emptyset$. Then

$$
\begin{gathered}
\mu(\emptyset)=\emptyset, \text { i.e. } \\
\mu\left(J_{M}\left(\mu, \mu^{\prime}\right)\right)=J_{W}\left(\mu, \mu^{\prime}\right)
\end{gathered}
$$

so the closing property holds.

Given a marriage model $(M, W, R)$, we say that $R$ is a dichotomous profile of preferences where each man evaluates each woman as acceptable or unacceptable: acceptable partners are indifferent to each other. 
The following Proposition shows that if we consider the marriage model with indifferences $(M, W, R)$ such that $R$ is a dichotomous profile of preferences then the stable matching set satisfies the closing property.

Proposition 3 Let $(M, W, R)$ be a marriage model with indifferences. If $R$ is a dichotomous profile of preferences then the stable matching set $S(R)$ satisfies the closing property.

Proof Let $\mu, \mu^{\prime} \in S(R)$. If $J_{M}\left(\mu, \mu^{\prime}\right)=J_{W}\left(\mu, \mu^{\prime}\right)=\emptyset$ then the closing property holds. We assume that $J_{W}\left(\mu, \mu^{\prime}\right) \neq \emptyset$. We will prove that

$$
\mu\left(J_{M}\left(\mu, \mu^{\prime}\right)\right)=J_{W}\left(\mu, \mu^{\prime}\right) .
$$

Firstly, we show that $J_{W}\left(\mu, \mu^{\prime}\right) \subseteq \mu\left(J_{M}\left(\mu, \mu^{\prime}\right)\right)$. Let $w \in J_{W}\left(\mu, \mu^{\prime}\right)$, we have $\mu(w) I_{w} \mu^{\prime}(w)$. Since $\mu(w) \neq \mu^{\prime}(w)$ there exist $m$ and $m^{\prime}$ such that

$$
m=\mu(w) I_{w} \mu^{\prime}(w)=m^{\prime} .
$$

By definition of matching $\mu(m)=w$. Since $\mu \in I R(R), \mu(m)=w$ and $\mu^{\prime}(m)$ are acceptable partners for $m$. Hence, since $R$ is a dichotomous profile of preferences

$$
w=\mu(m) I_{m} \mu^{\prime}(m)
$$

i.e. $w=\mu(m) \in \mu\left(J_{M}\left(\mu, \mu^{\prime}\right)\right)$.

Now, we show that $\mu\left(J_{M}\left(\mu, \mu^{\prime}\right)\right) \subseteq J_{W}\left(\mu, \mu^{\prime}\right)$. Let $m \in J_{M}\left(\mu, \mu^{\prime}\right)$, we have $\mu(m) I_{m} \mu^{\prime}(m)$. Since $\mu(m) \neq \mu^{\prime}(m)$ there exist $w$ and $w^{\prime}$ such that

$$
w=\mu(m) I_{m} \mu^{\prime}(m)=w^{\prime} .
$$

Since $\mu \in I R(R), \mu(w)=m$ and $\mu^{\prime}(w)$ are acceptable partners for $w$. Hence, since $R$ is a dichotomous profile of preferences

$$
m=\mu(w) I_{w} \mu^{\prime}(w),
$$

i.e. $w \in J_{W}\left(\mu, \mu^{\prime}\right)$.

When preferences are strict or dichotomous, we have seen that the set of stable matchings satisfies the closing property. If we consider a matching model that is a "combination of both" (the set of agents can be divided into two disjoint subsets: the set of agents that has strict preferences and the set 
of agents that has dichotomous preferences), then the closing property also holds.

In order to prove the following Proposition we need Manlove's Lemma, see the Appendix for a more detailed explanation.

Proposition 4 Let $(M, W, R)$ be a marriage model with indifferences then $S S(R)$ satisfies the closing property.

See the Appendix, for a proof.

Proposition 5 Let $(M, W, R)$ be a marriage model with indifferences then $S S S(R)$ satisfies the closing property.

See the Appendix, for a proof.

\section{The lattice structure}

From now on, we assume that $S(R)$ satisfies the closing property (unless otherwise specified). We will formulate the results of this section for the set of men $M$, symmetrical results are valid for the set of women $W$. We define the operations $\left(\vee_{M}\right)$ and $\left(\wedge_{M}\right)$ over matchings in the indifference case. We proved that if stable matchings satisfy the closing property, these operations are well defined over equivalence classes of stable matchings. We define lowest upper bound and greatest lower bound between equivalence classes of stable matchings.

We consider the "pointing" function used in the marriage and college admissions models with strict preferences. Given two stable matchings $\mu_{1}$ and $\mu_{2}$, suppose we are letting men select the best woman assigned to them through $\mu_{1}$ and $\mu_{2}$. In this way, we define $\mu_{1} \vee_{M} \mu_{2}$ on $M \cup W$.

Definition 6 Given $\mu_{1}, \mu_{2} \in \mathcal{M}$ we define a pre-matching

$\mu_{1} \vee_{M} \mu_{2}: M \cup W \rightarrow M \cup W$ by

$$
\begin{aligned}
& \mu_{1} \vee_{M} \mu_{2}(m)= \begin{cases}\mu_{1}(m) & \text { if } \mu_{1} P_{m} \mu_{2} \\
\mu_{2}(m) & \text { if } \mu_{2} R_{m} \mu_{1}\end{cases} \\
& \mu_{1} \vee_{M} \mu_{2}(w)= \begin{cases}\mu_{1}(w) \text { if } \mu_{2} P_{w} \mu_{1} \\
\mu_{2}(w) \text { if } \mu_{1} R_{w} \mu_{2} .\end{cases}
\end{aligned}
$$


The operation $\mu_{1} \wedge_{M} \mu_{2}$ is defined symmetrically, giving $m$ the worst of $\mu_{1}(m)$ and $\mu_{2}(m)$, and giving $w$ the best of $\mu_{1}(w)$ and $\mu_{2}\left(w_{2}\right)$. Similarly we can define the pre-matchings $\mu_{1} \vee_{W} \mu_{2}$ and $\mu_{1} \wedge_{W} \mu_{2}$.

The lattice theorem for the marriage model, Knuth (1976) and the college admissions problem, Roth and Sotomayor (1990) say that $\left(S(P), R_{M}, \vee_{M}, \wedge_{M}\right)$ is a lattice on $S(P)$. To see that in marriage model with indifferences framework, $\left(S(R), R_{M}, \vee_{M}, \wedge_{M}\right)$ may not be a lattice on $S(R)$ consider Example 3 below.

Example 3 Let $M=\left\{m_{1}, m_{2}\right\}$ be the set of men and $W=\left\{w_{1}, w_{2}\right\}$ be the set of women. Consider the preference profile $R$ :

$$
\begin{array}{ll}
R_{m_{1}}:\left[w_{1}, w_{2}\right], m_{1} . & R_{w_{1}}: m_{1}, m_{2}, w_{1} . \\
R_{m_{2}}: w_{1}, w_{2}, m_{2} . & R_{w_{2}}: m_{2}, m_{1}, w_{2} .
\end{array}
$$

The set of stable matchings consists of the following two matchings:

$$
\mu_{1}=\left(\begin{array}{ll}
m_{1} & m_{2} \\
w_{2} & w_{1}
\end{array}\right) \text { and } \mu_{2}=\left(\begin{array}{ll}
m_{1} & m_{2} \\
w_{1} & w_{2}
\end{array}\right)
$$

Observe that

$$
\mu_{1} \vee_{M} \mu_{2}\left(m_{1}\right)=\mu_{2}\left(m_{1}\right)=w_{1}
$$

but

$$
\mu_{1} \vee_{M} \mu_{2}\left(w_{1}\right)=\mu_{1}\left(w_{1}\right)=m_{2} .
$$

Therefore, the pre-matching $\mu_{1} \vee_{M} \mu_{2}$ is not even matching. Since $\mu_{2} \vee_{M} \mu_{1}\left(m_{1}\right)=\mu_{1}\left(m_{1}\right)=w_{2}$, we have that the pre-matching $\mu_{1} \vee_{M} \mu_{2}$ is not commutative, i.e., $\mu_{1} \vee_{M} \mu_{2}\left(m_{1}\right) \neq \mu_{2} \vee_{M} \mu_{1}\left(m_{1}\right)$.

Moreover, since $J_{M}\left(\mu_{1}, \mu_{2}\right)=\left\{m_{1}\right\}$ and $\mu_{1}\left(J_{M}\left(\mu_{1}, \mu_{2}\right)\right)=\left\{w_{2}\right\}$ we have that $S(R)$ does not satisfy the closing property.

The next Lemma shows that if $\mu_{1}$ and $\mu_{2}$ are stable matchings, then the pre-matchings $\mu_{1} \vee_{M} \mu_{2}$ and $\mu_{1} \wedge_{M} \mu_{2}$ are stable matchings.

Lemma 3 Let $(M, W, R)$ be a marriage model with indifferences. Let $\mu_{1}, \mu_{2} \in S(R)$ then

1. $\mu_{1} \vee_{M} \mu_{2} \in S(R)$.

2. $\mu_{1} \wedge_{M} \mu_{2} \in S(R)$. 
Proof Let $\mu_{1}, \mu_{2} \in S(R)$.

1. We prove that $\mu_{1} \vee_{M} \mu_{2}$ is a matching, by showing that $\mu_{1} \vee_{M} \mu_{2}(m)=w$ if and only if $\mu_{1} \vee_{M} \mu_{2}(w)=m$.

$\Longrightarrow)$ Suppose without loss of generality, that $\mu_{1} \vee_{M} \mu_{2}(m)=\mu_{2}(m)=w$. By definition of $\mu_{1} \vee_{M} \mu_{2}$, we have that

$$
w=\mu_{2}(m) R_{m} \mu_{1}(m)
$$

hence

$$
w=\mu_{2}(m) I_{m} \mu_{1}(m) \quad \text { or } \quad w=\mu_{2}(m) P_{m} \mu_{1}(m) .
$$

If

$$
w=\mu_{2}(m) I_{m} \mu_{1}(m)
$$

then $m \in J_{M}\left(\mu_{1}, \mu_{2}\right)$. By the closing property, we have that $\mu_{2}(m)=w \in J_{W}\left(\mu_{1}, \mu_{2}\right)$. This means that $\mu_{2}(w) I_{w} \mu_{1}(w)$. By definition of $\mu_{1} \vee_{M} \mu_{2}$, we have that $\mu_{1} \vee_{M} \mu_{2}(w)=\mu_{2}(w)=m$, the result follows. If

$$
w=\mu_{2}(m) P_{m} \mu_{1}(m)
$$

suppose that $\mu_{2}(w) P_{w} \mu_{1}(w)$ then condition (3) implies that the pair $(m, w)$ blocks $\mu_{1}$. This contradicts the stability of $\mu_{1}$. If $\mu_{1}(w) I_{w} \mu_{2}(w)=m$, it means that $\mu_{2}(m)=w \in J_{W}\left(\mu_{1}, \mu_{2}\right)$; by the closing property, we have that $m \in J_{M}\left(\mu_{1}, \mu_{2}\right)$. This contradicts $(3)$. Then $\mu_{1}(w) P_{w} \mu_{2}(w)$, by definition of $\mu_{1} \vee_{M} \mu_{2}$ we have that $\mu_{1} \vee_{M} \mu_{2}(w)=\mu_{2}(w)=m$. The result follows. The same reasoning applies to the case $\mu_{1} \vee_{M} \mu_{2}(m)=\mu_{1}(m)=w$.

$\Longleftarrow)$ To this purpose, we define the following sets:

$$
M^{\prime}=\left\{m: \mu_{1} \vee_{M} \mu_{2}(m) \in W\right\} \quad \text { and } \quad W^{\prime}=\left\{w: \mu_{1} \vee_{M} \mu_{2}(w) \in M\right\}
$$

We next claim $W^{\prime}=\mu_{1} \vee_{M} \mu_{2}\left(M^{\prime}\right)$. For this,

(a) $\mu_{1} \vee_{M} \mu_{2}\left(M^{\prime}\right) \subset W^{\prime}$ and $\# \mu_{1} \vee_{M} \mu_{2}\left(M^{\prime}\right) \leq \# W^{\prime}$. Let $m^{\prime} \in M^{\prime}$ then there exists $w \in W$ such that $\mu_{1} \vee_{M} \mu_{2}\left(m^{\prime}\right)=w$. By the previous proof, $\mu_{1} \vee_{M} \mu_{2}(w)=m^{\prime}$. This means that $w \in W^{\prime}$.

(b) $\mu_{1} \vee_{M} \mu_{2}$ is an injective function. To check that is a function, we prove that for each element of domain, there is a single image. This follows from let $m \in M$ we have that $\mu_{2} R_{m} \mu_{1}$ or $\mu_{1} P_{m} \mu_{2}$.

To establish that is an injective function, suppose that $\mu_{1} \vee_{M} \mu_{2}(m)=\mu_{1} \vee_{M} \mu_{2}(m)=w$. By the previous proof, $\mu_{1} \vee_{M} \mu_{2}(w)=m=m^{\prime}$. Consider $\mu_{1} \vee_{M} \mu_{2} \mid M^{\prime}$ is a function bijective hence $\# \mu_{1} \vee_{M} \mu_{2}\left(M^{\prime}\right)=\# M^{\prime}$. 
(c) $\# \mu_{1}\left(W^{\prime}\right)=\# W^{\prime}$. By definition of matching $\mu_{1}$ is an injective function. Considering $\mu_{1} \mid W^{\prime}$ is a bijective function therefore $\# \mu_{1}\left(W^{\prime}\right)=\# W^{\prime}$.

Items a, b, and c imply that

$$
\# W^{\prime}=\# \mu_{1}\left(W^{\prime}\right) \leq \# M^{\prime}=\# \mu_{1} \vee_{M} \mu_{2}\left(M^{\prime}\right) \leq \# W^{\prime}
$$

hence,

$$
\# W^{\prime}=\# \mu_{1} \vee_{M} \mu_{2}\left(M^{\prime}\right) .
$$

Item (a) and condition (4) imply that

$$
W^{\prime}=\mu_{1} \vee_{M} \mu_{2}\left(M^{\prime}\right) .
$$

We are now in position to show that for all $w \in W$, if $\mu_{1} \vee_{M} \mu_{2}(w)=m$ then $\mu_{1} \vee_{M} \mu_{2}(m)=w$. If $w \notin W^{\prime}$ then $\mu_{1} \vee_{M} \mu_{2}(w)=w$. This contradicts that $\mu_{1} \vee_{M} \mu_{2}(m)=w$. So $w \in W^{\prime}$ and $\mu_{1} \vee_{M} \mu_{2}(w)=m$ then there exists $m^{\prime} \in M^{\prime}$ such that $\mu_{1} \vee_{M} \mu_{2}\left(m^{\prime}\right)=w$ then $\mu_{1} \vee_{M} \mu_{2}(w)=m^{\prime}=m$ and the result follows.

Now, we will show the stability. Suppose that there exist a pair $(m, w)$ such that

$$
m P_{w} \mu_{1} \vee_{M} \mu_{2}(w) \text { and } w P_{m} \mu_{1} \vee_{M} \mu_{2}(m) .
$$

We consider the following cases:

Case i. $\mu_{1} \vee_{M} \mu_{2}(w)=\mu_{1}(w)\left(\mu_{1} \vee_{M} \mu_{2}(w)=\mu_{2}(w)\right)$ and

$\mu_{1} \vee_{M} \mu_{2}(m)=\mu_{1}(m)\left(\mu_{1} \vee_{M} \mu_{2}(m)=\mu_{2}(m)\right)$ so (5) contradicts $\mu_{1} \in S(R)$ $\left(\mu_{2} \in S(R)\right)$.

Case ii. $\mu_{1} \vee_{M} \mu_{2}(w)=\mu_{1}(w)$ and $\mu_{1} \vee_{M} \mu_{2}(m)=\mu_{2}(m)$. By (5) and definition of $\mu_{1} \vee_{M} \mu_{2}$ we have that

$$
m P_{w} \mu_{1}(w) \text { and } \quad w P_{m} \mu_{2}(m) R_{m} \mu_{1}(m)
$$

hence the pair $(m, w)$ blocks $\mu_{1}$. This contradicts the stability of $\mu_{1}$.

Case iii. $\mu_{1} \vee_{M} \mu_{2}(w)=\mu_{2}(w)$ and $\mu_{1} \vee_{M} \mu_{2}(m)=\mu_{1}(m)$. Like Case ii, we can contradict the stability of $\mu_{2}$.

2. That $\mu_{1} \wedge_{M} \mu_{2}$ is a stable matching follows from a similar argument.

The following Lemma asserts that $\left[\mu_{1} \vee_{M} \mu_{2}\right]$ and $\left[\mu_{1} \wedge_{M} \mu_{2}\right]$ are independent of the particular choices of representatives of the equivalence classes $\left[\mu_{1}\right]$ and $\left[\mu_{2}\right]$. 
Lemma 4 Let $(M, W, R)$ be a marriage model with indifferences. Let $\mu_{1}, \mu_{2} \in S(R)$ then for all $\mu_{i}^{\prime} \in\left[\mu_{i}\right], i=1,2$

1. $\left(\mu_{1}^{\prime} \vee_{M} \mu_{2}^{\prime}\right) I\left(\mu_{1} \vee_{M} \mu_{2}\right)$, i.e. $\mu_{1}^{\prime} \vee_{M} \mu_{2}^{\prime} \in\left[\mu_{1} \vee_{M} \mu_{2}\right]$.

2. $\left(\mu_{1}^{\prime} \wedge_{M} \mu_{2}^{\prime}\right) I\left(\mu_{1} \wedge_{M} \mu_{2}\right)$, i.e. $\mu_{1}^{\prime} \wedge_{M} \mu_{2}^{\prime} \in\left[\mu_{1} \wedge_{M} \mu_{2}\right]$.

Proof Let $\mu_{1}, \mu_{2} \in S(R)$ and $\mu_{i}^{\prime} \in\left[\mu_{i}\right], i=1,2$.

1. We claim that $\left(\mu_{1}^{\prime} \vee_{M} \mu_{2}^{\prime}\right) I\left(\mu_{1} \vee_{M} \mu_{2}\right)$. To check it, suppose that $\mu_{1} \vee_{M} \mu_{2}(m)=\mu_{1}(m)$. By definition of $\mu_{1} \vee_{M} \mu_{2}$ and $\mu_{i}^{\prime} \in\left[\mu_{i}^{\prime}\right], i=1,2$ we have that

$$
\mu_{1}^{\prime}(m) I_{m} \mu_{1}(m) P_{m} \mu_{2}(m) I_{m} \mu_{2}^{\prime}(m) .
$$

By Lemma $1 \mu_{1}^{\prime}(m) P_{m} \mu_{2}^{\prime}(m)$. Hence,

$$
\mu_{1}^{\prime} \vee_{M} \mu_{2}^{\prime}(m)=\mu_{1}^{\prime}(m) I_{m} \mu_{1}(m)=\mu_{1} \vee_{M} \mu_{2}(m) .
$$

This means that $M \subseteq J_{M}\left(\mu_{1}^{\prime}, \mu_{2}^{\prime}\right)$. Suppose that there exist $w \in W$ such that $w \notin J_{W}\left(\mu_{1}^{\prime}, \mu_{2}^{\prime}\right)$. Rational individuality of $\mu_{1}^{\prime}$ and $\mu_{2}^{\prime}$ implies that there exist $m \in M$ such that either

$$
m=\mu_{1}^{\prime}(w) P_{w} \mu_{2}^{\prime}(w) \text { or } m=\mu_{2}^{\prime}(w) P_{w} \mu_{1}^{\prime}(w) .
$$

This contradicts the fact that $m \in J_{M}\left(\mu_{1}^{\prime}, \mu_{2}^{\prime}\right)$. The same reasoning applies to the case that $\mu_{1} \vee_{M} \mu_{2}(m)=\mu_{2}(m)$.

2. The proof is similar to 1 .

In order to establish the existence of a lattice structure, we need to define two operations $\vee_{M}$ and $\wedge_{M}$ on $S(R) / \sim$.

Definition 7 Given $\left[\mu_{1}\right],\left[\mu_{2}\right] \in S(R) / \sim$ we define

$$
\begin{aligned}
& {\left[\mu_{1}\right] \vee_{M}\left[\mu_{2}\right]=\left[\mu_{1} \vee_{M} \mu_{2}\right]} \\
& {\left[\mu_{1}\right] \wedge_{M}\left[\mu_{2}\right]=\left[\mu_{1} \wedge_{M} \mu_{2}\right] .}
\end{aligned}
$$

Similarly, we can define $\left[\mu_{1}\right] \vee_{W}\left[\mu_{2}\right]$ and $\left[\mu_{1}\right] \wedge_{W}\left[\mu_{2}\right]$. From Lemma 3 and 4 the binary operations $\vee_{M}$ and $\wedge_{M}$ are well defined over $S(R) / \sim$.

In our context, we can define a lattice on $S(R) / \sim$ if there exists a partial order $R_{M}$ and two operations $\vee_{M}$ and $\wedge_{M}$ on $S(R) / \sim$ such that for all $\left[\mu_{1}\right],\left[\mu_{2}\right],[\nu] \in S(R) / \sim$ the following properties hold: 
(1) $\left[\mu_{1}\right] \vee_{M}\left[\mu_{2}\right] \in S(R) / \sim$.

(2) $\left[\mu_{1}\right] \wedge_{M}\left[\mu_{2}\right] \in S(R) / \sim$.

(3) $\left[\mu_{1}\right] \vee_{M}\left[\mu_{2}\right] R_{M}\left[\mu_{1}\right]$ and $\left[\mu_{1}\right] \vee_{M}\left[\mu_{2}\right] R_{M}\left[\mu_{2}\right]$.

(4) $\left[\mu_{1}\right] R_{M}\left[\mu_{1}\right] \wedge_{M}\left[\mu_{2}\right]$ and $\left[\mu_{2}\right] R_{M}\left[\mu_{1}\right] \wedge_{M}\left[\mu_{2}\right]$.

(5) $[\nu] R_{M}\left[\mu_{1}\right]$ and $[\nu] R_{M}\left[\mu_{2}\right]$ then $[\nu] R_{M}\left[\mu_{1}\right] \vee_{M}\left[\mu_{2}\right]$.

(6) $\left[\mu_{1}\right] R_{M}[\nu]$ and $\left[\mu_{2}\right] R_{M}[\nu]$ then $\left[\mu_{1}\right] \wedge_{M}\left[\mu_{2}\right] R_{M}[\nu]$.

Conditions (1) and (2) say that $\vee_{M}$ and $\wedge_{M}$ are binary operations on $S(R) / \sim$. Conditions (3)-(6) say that $\left[\mu_{1}\right] \vee_{M}\left[\mu_{2}\right]$ and $\left[\mu_{1}\right] \wedge_{M}\left[\mu_{2}\right]$ are, respectively, the least upper bound and the greatest lower bound of $\left[\mu_{1}\right]$ and $\left[\mu_{2}\right]$ according to the partial order $R_{M}$. The quadruple $\left(S(R) / \sim, R_{M}, \vee_{M}, \wedge_{M}\right)$ is called a lattice on $S(R) / \sim$.

We are now in position to present our main result of this section.

Theorem 1 Let $(M, W, R)$ be a marriage model with indifferences. The quadruple $\left(S(R) / \sim, R_{M}, \vee_{M}, \wedge_{M}\right)$ is a lattice over $S(R) / \sim$.

Proof Let $\mu_{i}^{\prime} \in\left[\mu_{i}\right], i=1,2$.

(1) By definition of $\left[\mu_{1}\right] \vee_{M}\left[\mu_{2}\right]$ we have $\left[\mu_{1}\right] \vee_{M}\left[\mu_{2}\right]=\left[\mu_{1} \vee_{M} \mu_{2}\right]$. From Lemma 3 and $4, \mu_{1}^{\prime} \vee_{M} \mu_{2}^{\prime} \in S(R)$ and $\left[\mu_{1} \vee_{M} \mu_{2}\right]$ is independent of the choice of the representative. This means that $\left[\mu_{1} \vee_{M} \mu_{2}\right] \in S(R) / \sim$, i.e.

$\left[\mu_{1}\right] \vee_{M}\left[\mu_{2}\right] \in S(R) / \sim$.

(2) This can be proved by arguments similar to (1).

(3) We have that $\left[\mu_{1}\right] \vee_{M}\left[\mu_{2}\right]=\left[\mu_{1} \vee_{M} \mu_{2}\right]$. By definition of $\mu_{1} \vee_{M} \mu_{2}$,

$$
\mu_{1}^{\prime} \vee_{M} \mu_{2}^{\prime} R_{M} \mu_{1}^{\prime} \text { and } \mu_{1}^{\prime} \vee_{M} \mu_{2}^{\prime} R_{M} \mu_{2}^{\prime} \text {. }
$$

Then,

$$
\left[\mu_{1} \vee_{M} \mu_{2}\right] R_{M}\left[\mu_{1}\right] \text { and }\left[\mu_{1} \vee_{M} \mu_{2}\right] R_{M}\left[\mu_{2}\right]
$$

Therefore,

$$
\left[\mu_{1}\right] \vee_{M}\left[\mu_{2}\right] R_{M}\left[\mu_{1}\right] \text { and }\left[\mu_{1}\right] \vee_{M}\left[\mu_{2}\right] R_{M}\left[\mu_{2}\right]
$$


(4) This can be proved by arguments similar to (3).

(5) Let $[\nu] R_{M}\left[\mu_{1}\right]$ and $[\nu] R_{M}\left[\mu_{2}\right]$. Let's assume that $\overline{[\nu] R_{M}\left[\mu_{1}\right] \vee_{M}\left[\mu_{2}\right]}$. By definition of $\left[\mu_{1}\right] \vee_{M}\left[\mu_{2}\right]$, we have that $[\nu] R_{M}\left[\mu_{1} \vee_{M} \mu_{2}\right]$. Then, there exist $j \in M$ such that

$$
\left[\mu_{1} \vee_{M} \mu_{2}\right] P_{j}[\nu] R_{j}\left[\mu_{1}\right]
$$

Definition of $\mu_{1} \vee_{M} \mu_{2}$ and condition (6) imply that

$$
\mu_{1}^{\prime} \vee_{M} \mu_{2}^{\prime} P_{j} \nu^{\prime} R_{j} \mu_{1}^{\prime}
$$

for all $\mu_{i}^{\prime} \in\left[\mu_{i}\right]$ and $\nu^{\prime} \in[\nu]$. Hence, $\mu_{1}^{\prime} \vee_{M} \mu_{2}^{\prime} \neq \mu_{1}^{\prime}$; i.e. $\mu_{1}^{\prime} \vee_{M} \mu_{2}^{\prime}=\mu_{2}^{\prime}$ and $\mu_{2}^{\prime} P_{j} \nu^{\prime}$ which contradicts $[\nu] R_{M}\left[\mu_{2}\right]$. Therefore $[\nu] R_{M}\left[\mu_{1} \vee_{M} \mu_{2}\right]$.

(6) The proof results similar to (5).

Let $S S(R) \subseteq S(R),\left(S S(R) / \sim, R_{M}, \vee_{M}, \wedge_{M}\right)$ is a sublattice of a lattice $\left(S(R) / \sim, R_{M}, \vee_{M}, \wedge_{M}\right)$ if for all $\left[\mu_{1}\right],\left[\mu_{2}\right] \in S S(R) / \sim$ the following properties hold:

(1) $\left[\mu_{1}\right] \vee_{M}\left[\mu_{2}\right] \in S S(R) / \sim$.

(2) $\left[\mu_{1}\right] \wedge_{M}\left[\mu_{2}\right] \in S S(R) / \sim$.

The following Theorem asserts that $\left(S S(R) / \sim, R_{M}, \vee_{M}, \wedge_{M}\right)$ is a sublattice of the $\left(S(R) / \sim, \preceq, \vee_{M}, \wedge_{M}\right)$.

Theorem 2 Let $(M, W, R)$ be a marriage model with indifferences. The quadruple $\left(S S(R) / \sim, R_{M}, \vee_{M}, \wedge_{M}\right)$ is a sublattice of the $\left(S(R) / \sim, R_{M}, \vee_{M}, \wedge_{M}\right)$.

Proof Let $\mu_{1}, \mu_{2} \in S S(R) \subseteq S(R)$.

(1) By Lemma 3, $\mu_{1} \vee_{M} \mu_{2} \in S(R)$. Let's suppose that there exist $\mu_{i}^{\prime} \in S S(R)$ and $\mu_{i}^{\prime} \in\left[\mu_{i}\right]$ for $i=1,2$, such that $\mu_{1}^{\prime} \vee_{M} \mu_{2}^{\prime} \notin S S(R)$. Then, there exists a strongly blocking pair $(m, w)$,

$$
w P_{m} \mu_{1}^{\prime} \vee_{M} \mu_{2}^{\prime}(m) \text { and } m R_{w} \mu_{1}^{\prime} \vee_{M} \mu_{2}^{\prime}(w) .
$$


If $m P_{w} \mu_{1}^{\prime} \vee_{M} \mu_{2}^{\prime}(w)$ then the pair $(m, w)$ blocks $\mu_{1}^{\prime} \vee_{M} \mu_{2}^{\prime}$. This contradicts $\mu_{1}^{\prime} \vee_{M} \mu_{2}^{\prime} \in S(R)$. So,

$$
w P_{m} \mu_{1}^{\prime} \vee_{M} \mu_{2}^{\prime}(m) \text { and } m I_{w} \mu_{1}^{\prime} \vee_{M} \mu_{2}^{\prime}(w) .
$$

Suppose that $\mu_{1}^{\prime} \vee_{M} \mu_{2}^{\prime}(w)=\mu_{1}^{\prime}(w)$. Condition (7) and definition of $\mu_{1}^{\prime} \vee_{M} \mu_{2}^{\prime}$ imply that

$$
w P_{m} \mu_{1}^{\prime} \vee_{M} \mu_{2}^{\prime}(m) R_{m} \mu_{1}^{\prime}(m) \text { and } m I_{w} \mu_{1}^{\prime}(w) .
$$

Then, the pair $(m, w)$ strongly blocks $\mu_{1}^{\prime}$. This contradicts $\mu_{1}^{\prime} \in S S(R)$. So $\mu_{1}^{\prime} \vee_{M} \mu_{2}^{\prime}(w)=\mu_{2}^{\prime}(w)$, condition (7) and definition of $\mu_{1}^{\prime} \vee_{M} \mu_{2}^{\prime}$ imply that

$$
w P_{m} \mu_{1}^{\prime} \vee_{M} \mu_{2}^{\prime}(m) R_{m} \mu_{2}^{\prime}(m) \text { and } m I_{w} \mu^{\prime}(w) \text {. }
$$

Then, the pair $(m, w)$ strongly blocks $\mu_{2}^{\prime}$. This contradicts $\mu_{2}^{\prime} \in S S(R)$. Contradiction comes from assuming that $\mu_{1}^{\prime} \vee_{M} \mu_{2}^{\prime} \notin S S(R)$. Hence $\mu_{1}^{\prime} \vee_{M} \mu_{2}^{\prime} \in S S(R)$ and $\left[\mu_{1}\right] \vee_{M}\left[\mu_{2}\right] \in S S(R) / \sim$.

(2) The proof is similar to (1).

The following example shows that $\left(S S(R) / \sim, R_{M}, \vee_{M}, \wedge_{M}\right)$ is a strict sublattice of the $\left(S(R) / \sim, R_{M}, \vee_{M}, \wedge_{M}\right)$.

Example 4 Let $M=\left\{m_{1}, m_{2}, m_{3}, m_{4}\right\}$ be the set of men and $W=\left\{w_{1}, w_{2}, w_{3}, w_{4}\right\}$ be the set of women. Consider the preference profile $R$ :

$$
\begin{array}{ll}
R_{m_{1}}: w_{1},\left[w_{2}, w_{3}\right], m_{1} . & R_{w_{1}}: m_{3},\left[m_{1}, m_{2}\right], w_{1} . \\
R_{m_{2}}: w_{1}, w_{4}, m_{2} . & R_{w_{2}}:\left[m_{1}, m_{4}\right], w_{2} . \\
R_{m_{3}}: w_{4}, w_{1}, m_{3} . & R_{w_{3}}:\left[m_{1}, m_{4}\right], w_{3} . \\
R_{m_{4}}:\left[w_{2}, w_{3}\right], m_{4} . & R_{w_{4}}: m_{2}, m_{3}, w_{4} .
\end{array}
$$

The set of stable matchings consists of the following four matchings:

$$
\begin{aligned}
\mu_{1} & =\left(\begin{array}{llll}
m_{1} & m_{2} & m_{3} & m_{4} \\
w_{2} & w_{1} & w_{4} & w_{3}
\end{array}\right), \quad \mu_{2}=\left(\begin{array}{llll}
m_{1} & m_{2} & m_{3} & m_{4} \\
w_{3} & w_{1} & w_{4} & w_{2}
\end{array}\right) \\
\mu_{3} & =\left(\begin{array}{llll}
m_{1} & m_{2} & m_{3} & m_{4} \\
w_{2} & w_{4} & w_{1} & w_{3}
\end{array}\right) \text { and } \mu_{4}=\left(\begin{array}{llll}
m_{1} & m_{2} & m_{3} & m_{4} \\
w_{3} & w_{4} & w_{1} & w_{2}
\end{array}\right) .
\end{aligned}
$$

The reader can check that $S(R)$ satisfies the closing property. Observe that $S S(R)=\left\{\mu_{3}, \mu_{4}\right\}$. Since $\mu_{1} I_{i} \mu_{2}$ and $\mu_{3} I_{i} \mu_{4}$ for all $i \in M \cup W$. It follows that $\left[\mu_{1}\right]=\left[\mu_{2}\right]$ and $\left[\mu_{3}\right]=\left[\mu_{4}\right]$. Thus,

$$
S S(R) / \sim=\left\{\left[\mu_{3}\right]\right\} \varsubsetneqq\left\{\left[\mu_{1}\right],\left[\mu_{3}\right]\right\}=S(R) / \sim .
$$


Note that the preference is not strict or dichotomous, neither is a combination of both. Nevertheless it satisfies the closing property.

\section{Conflict of interests}

In the marriage model with strict preferences, the Decomposition Lemma was originally proved by Knuth (1976). Next, we will prove the Decomposition Lemma in our context.

Given two matchings $\mu_{1}$ and $\mu_{2}$, we define the following sets:

$$
M\left(\mu_{2}\right)=\left\{m \in M: \mu_{2} P_{m} \mu_{1}\right\} \quad \text { and } \quad W\left(\mu_{1}\right)=\left\{w \in W: \mu_{1} P_{w} \mu_{2}\right\} .
$$

Lemma 5 (Decomposition Lemma) Let $(M, W, R)$ be a marriage model with indifferences. Let $\mu_{1}, \mu_{2} \in S(R)$ such that $\left[\mu_{1}\right] \neq\left[\mu_{2}\right]$ then for all $\mu_{i}^{\prime} \in\left[\mu_{i}\right], i=1,2$

$$
\begin{aligned}
& \mu_{2}^{\prime}\left(M\left(\mu_{2}^{\prime}\right)\right)=W\left(\mu_{1}^{\prime}\right)\left(\text { or } \mu_{2}\left(W\left(\mu_{1}^{\prime}\right)\right)=M\left(\mu_{2}^{\prime}\right)\right) \\
& \mu_{1}^{\prime}\left(W\left(\mu_{1}^{\prime}\right)\right)=M\left(\mu_{2}^{\prime}\right)\left(\text { or } \mu_{1}^{\prime}\left(M\left(\mu_{2}^{\prime}\right)\right)=W\left(\mu_{1}^{\prime}\right)\right) .
\end{aligned}
$$

Proof Let $\mu_{i}^{\prime}$ be stable matchings such that $\mu_{i}^{\prime} \in\left[\mu_{i}\right], i=1,2$. Since $\left[\mu_{1}\right] \neq\left[\mu_{2}\right]$ we have that either $M\left(\mu_{2}^{\prime}\right) \neq \emptyset$ or $M\left(\mu_{1}^{\prime}\right) \neq \emptyset$. We assume that $M\left(\mu_{2}^{\prime}\right) \neq \emptyset$. First, we will show that

$$
\mu_{2}^{\prime}\left(M\left(\mu_{2}^{\prime}\right)\right) \subseteq W\left(\mu_{1}^{\prime}\right)
$$

To check it, given $m \in M\left(\mu_{2}^{\prime}\right)$, by definition of $M\left(\mu_{2}^{\prime}\right)$ and rational individuality of $\mu_{1}^{\prime}$, there exists $w$ such that

$$
w=\mu_{2}^{\prime}(m) P_{m} \mu_{1}^{\prime}(m) R_{m} m
$$

If

$$
m=\mu_{2}^{\prime}(w) P_{w} \mu_{1}^{\prime}(w),
$$

then conditions (9) and (10) imply that the pair $(m, w)$ blocks $\mu_{1}^{\prime}$. This contradicts $\mu_{1}^{\prime} \in S(R)$. Hence,

$$
\mu_{1}^{\prime}(w) R_{w} \mu_{2}^{\prime}(w)
$$


note that (9) says that $m \notin J_{M}\left(\mu_{1}^{\prime}, \mu_{2}^{\prime}\right)$. Then by the closing property $\mu_{2}^{\prime}(m)=w \notin J_{W}\left(\mu_{1}^{\prime}, \mu_{2}^{\prime}\right)$. So

$$
\mu_{1}^{\prime}(w) P_{w} \mu_{2}^{\prime}(w)
$$

This means that $w \in W\left(\mu_{1}^{\prime}\right)$. This proves condition (8).

By definition of matching $\mu_{2}^{\prime}$ is an injective function. This together with condition (8) imply that

$$
\# M\left(\mu_{2}^{\prime}\right) \leq \# W\left(\mu_{1}^{\prime}\right) .
$$

Similarly,

$$
\mu_{1}^{\prime}\left(W\left(\mu_{1}^{\prime}\right)\right) \subseteq M\left(\mu_{2}^{\prime}\right),
$$

and

$$
\# W\left(\mu_{1}^{\prime}\right) \leq \# M\left(\mu_{2}^{\prime}\right) .
$$

Conditions (11) and (13) imply that

$$
\# M\left(\mu_{2}^{\prime}\right) \leq \# W\left(\mu_{1}^{\prime}\right) \leq \# M\left(\mu_{2}^{\prime}\right)
$$

Hence,

$$
\# M\left(\mu_{2}^{\prime}\right)=\# W\left(\mu_{1}^{\prime}\right) .
$$

This together with condition (8) imply that

$$
\mu_{2}^{\prime}\left(M\left(\mu_{2}^{\prime}\right)\right)=W\left(\mu_{1}^{\prime}\right) .
$$

Definition of $\mu_{2}^{\prime}$ and (15) imply that

$$
\mu_{2}\left(W\left(\mu_{1}^{\prime}\right)\right)=M\left(\mu_{2}^{\prime}\right) .
$$

Conditions (12) and (14) imply that

$$
\mu_{1}^{\prime}\left(W\left(\mu_{1}^{\prime}\right)\right)=M\left(\mu_{2}^{\prime}\right) .
$$

Definition of $\mu_{1}^{\prime}$ and (16) imply that

$$
\mu_{1}^{\prime}\left(M\left(\mu_{2}^{\prime}\right)\right)=W\left(\mu_{1}^{\prime}\right) .
$$

Next, we see that agents on one side of the market have a common interest regarding the set of equivalences classes of stable matchings. If $\left[\mu_{2}\right]$ is a stable 
class that is weakly preferred to other stable class $\left[\mu_{1}\right]$ for all men then $\left[\mu_{1}\right]$ is weakly preferred to $\left[\mu_{2}\right]$ for all women. Formally,

Proposition 6 (Conflict of interests) Let $(M, W, R)$ be a marriage model with indifferences. Let $\left[\mu_{1}\right],\left[\mu_{2}\right] \in S(R) / \sim$. If $\left[\mu_{2}\right] R_{M}\left[\mu_{1}\right]$, then $\left[\mu_{1}\right] R_{W}\left[\mu_{2}\right]$.

Proof We assume that $\overline{\left[\mu_{1}\right] R_{W}\left[\mu_{2}\right]}$. Then there exist $\mu_{i}^{\prime} \in\left[\mu_{i}\right], i=1,2$ such that $\overline{\mu_{1}^{\prime} R_{W} \mu_{2}^{\prime}}$, i.e. there exists $w \in W$ such that

$$
m=\mu_{2}^{\prime}(w) P_{w} \mu_{1}^{\prime}(w)
$$

This means that $w \in W\left(\mu_{2}\right)$. By Lemma 5 we have that $m=\mu_{2}^{\prime}(w) \in M\left(\mu_{1}^{\prime}\right)$; i.e.

$$
\mu_{1}^{\prime}(m) P_{m} \mu_{2}^{\prime}(w)=m .
$$

This contradicts $\left[\mu_{2}\right] R_{M}\left[\mu_{1}\right]$.

We conclude from Theorem 1 and Proposition 6 that $S(R) / \sim$ is a lattice under the common order of men $R_{M}$, dual to the common order of women.

\section{The rural hospital theorem}

In this section we generalize the rural hospital theorem under indifferences. In order to prove the rural hospital theorem, we assume that $S(R)$ satisfies the closing property.

The following example shows that if $S(R)$ does not satisfy the closing property then the rural hospital theorem is not valid.

Example 5 Let $M=\left\{m_{1}\right\}$ be the set of men and $W=\left\{w_{1}, w_{2}\right\}$ be the set of women. Consider the preference profile $R$ :

$$
\begin{aligned}
R_{m_{1}}:\left[w_{1}, w_{2}\right], m_{1} . & R_{w_{1}}: m_{1}, w_{1} . \\
& R_{w_{2}}: m_{1}, w_{2} .
\end{aligned}
$$

The set of stable matchings consists of the following two matchings:

$$
\mu_{1}=\left(\begin{array}{ll}
w_{1} & w_{2} \\
m_{1} & w_{2}
\end{array}\right) \text { and } \mu_{2}=\left(\begin{array}{cc}
w_{1} & w_{2} \\
w_{1} & m_{1}
\end{array}\right)
$$


We have that $S(R)$ does not satisfy the closing property because

$$
J_{M}\left(\mu_{1}, \mu_{2}\right) \text { and } J_{W}\left(\mu_{1}, \mu_{2}\right)=\emptyset .
$$

On the other hand

$$
\mu_{1}\left(J_{M}\left(\mu_{1}, \mu_{2}\right)\right)=\left\{w_{1}\right\} \neq J_{W}\left(\mu_{1}, \mu_{2}\right) .
$$

Since $\mu_{1}\left(w_{2}\right)=w_{2}$ and $\mu_{2}\left(w_{2}\right)=m_{1}$, we see that the rural hospital theorem is not valid.

Irving, Manlove and Scott (2000) studied the indifferences in hospital and residents model and they proved that if the super stable set is not empty the rural hospital theorem is valid over all stable matchings. If the stable matching set satisfies the closing property then the rural hospital theorem is valid, even when the super stable matching set is empty. See the example 1 again below.

Example 1 Let $M=\left\{m_{1}, m_{2}\right\}$ be the set of men and $W=\left\{w_{1}, w_{2}\right\}$ be the set of women. Consider the preference profile $R$ :

$$
\begin{array}{cc}
R_{m_{1}}:\left[w_{1}, w_{2}\right], m_{1} . & R_{w_{1}}:\left[m_{1}, m_{2}\right], w_{1} . \\
R_{m_{2}}:\left[w_{1}, w_{2}\right], m_{2} . & R_{w_{2}}:\left[m_{2}, m_{1}\right], w_{2} .
\end{array}
$$

We have seen that, the set of stable matchings consists of the following two matchings:

$$
\mu_{1}=\left(\begin{array}{ll}
m_{1} & m_{2} \\
w_{1} & w_{2}
\end{array}\right) \text { and } \mu_{2}=\left(\begin{array}{ll}
m_{1} & m_{2} \\
w_{2} & w_{1}
\end{array}\right)
$$

We have that $S(R)$ satisfies the closing property because

$$
\begin{gathered}
J_{M}\left(\mu_{1}, \mu_{2}\right)=M \text { and } J_{W}\left(\mu_{1}, \mu_{2}\right)=W \\
\mu_{1}\left(J_{M}\left(\mu_{1}, \mu_{2}\right)\right)=\mu_{2}\left(J_{M}\left(\mu_{1}, \mu_{2}\right)\right)=J_{W}\left(\mu_{1}, \mu_{2}\right)=W .
\end{gathered}
$$

We have $\mu_{1} I \mu_{2}$. So the super stable matching set is empty. Since there are not single agents in $\mu_{1}$ and $\mu_{2}$, the rural hospital theorem is valid.

Theorem 3 Let $(M, W, R)$, be a marriage model with indifferences. If $S(R)$ satisfies the closing property then the set of people who are single is the same for all stable matchings. 
Proof Let $\mu_{1}$ and $\mu_{2}$ be two stable matchings, and $\mu_{i}^{\prime} \in\left[\mu_{i}\right], i=1,2$. If $\left[\mu_{1}\right]=\left[\mu_{2}\right]$, the result follows from no indifferences to the single set assumption. Suppose that $\left[\mu_{1}\right] \neq\left[\mu_{2}\right]$. If

$$
w=\mu_{2}^{\prime}(m) I_{m} \mu_{1}^{\prime}(m)
$$

for all $m \in M$. By the closing property we have that $\mu_{1} I_{w} \mu_{2}$ for all $w \in W$. Hence $\left[\mu_{1}\right]=\left[\mu_{2}\right]$. Contradicts the assumption, then there exists $m \in M$ such that

$$
w=\mu_{2}^{\prime}(m) P_{m} \mu_{1}^{\prime}(m) \text { or } w=\mu_{1}^{\prime}(m) P_{m} \mu_{2}^{\prime}(m) .
$$

We assume without loss of generality that

$$
w=\mu_{2}^{\prime}(m) P_{m} \mu_{1}^{\prime}(m)=m .
$$

This means that $m \in M\left(\mu_{2}^{\prime}\right)$. By Lemma 5 we have that $M\left(\mu_{2}^{\prime}\right)=\mu_{1}^{\prime}\left(W\left(\mu_{1}^{\prime}\right)\right)$, and there exists $w^{*} \in W\left(\mu_{1}^{\prime}\right)$ such as $\mu_{1}^{\prime}\left(w^{*}\right)=m$ this contradicts $\mu_{1}^{\prime}(m)=m$.

If the strongly stable set is not empty by Proposition 4, the strongly stable set satisfies the closing property so the previous Theorem assures that the rural hospital theorem holds for all strongly stable matchings.

Corollary 1 Let $(M, W, R)$, be a marriage model with indifferences. If $S S(R) \neq \emptyset$ then the set of people who are single is the same for all strongly stable matchings. 


\section{Final remarks}

We hope this paper contributes to the literature by proposing a new sufficient condition, the closing property, which allows us to extend results from the marriage model with strict preferences to the marriage model with indifferences. In particular, we give a proof of the lattice structure over equivalence classes of the set of stable matchings. In addition, since the strongly stable matching set always satisfies the closing property we generalize the la-ttice structure over equivalence classes of the set of strongly stable matchings (Manlove 2002). The lattice over equivalence classes of strongly stable mat-chings is a sublattice of the lattice over equivalence classes of stable matchings. In addition, if the stable matching set satisfies the closing property we show the rural hospital theorem over stable matchings.

In the appendix, we show that effectively the closing property is not a necessary condition to the lattice structure of stable matching set. (See Example 6)

Although the closing property is defined on the matching set and not on the preferences, we show special preferences that satisfy it. Strict and dichotomous preferences satisfy the closing property. Moreover a matching model that is a "combination of both", strict and dichotomous preferences, also satisfies the closing property. Example 4, shows that there are more preferences than the strict and dichotomous preferences that satisfy the closing property. An open question is whether it is possible to characterize the closing property in terms of preferences of agents. This problem seems to be difficult to solve. To the best of our knowledge, there are no papers in the literature on matchings models with indifferences that provide characterizations about the preferences of the agents that allow obtaining results about the stable matchings set. 


\section{References:}

1. Bogomolnaia, A. and Moulin, H. (2004). "Random Matching Under Dichotomous Preferences", Econometrica, 72, 257-279.

2. Erdil, A. and Ergin, H. (2017). "Two-Sided Matching with Indifferences", Journal of Economic Theory, 171, 268-292.

3. Erdil, A. and Ergin, H. (2008). "What's the Matter with Tie-Breaking? Improvement Efficiency in School Choice", American Economic Review, 98, 669-89.

4. Irving, R. (1994). "Stable marriage and indifference", Discrete Applied Mathematics 48, 261-272.

5. Irving, R., Manlove D.F. and Scott, S. (2000). "The Hospitals/Residents Problem with Ties." In proceedings of ICALP'2000. Lecture Notes in Computer Science, 1851, 259-271. Springer-Verlag.

6. Knuth, D. (1976). Marriages Stables et leurs relations avec d'autres problèmes combinatoires, Les Presses de l'Université de Montréal, Montréal. Translated as Stable Marriage and Its Relation to Other Combinatorial Problems by Martin Goldstein, American Mathematical Society, Providence, Rhode Island, 1997, 253-255.

7. Manlove, D. (2002). "The Structure of Stable Marriage with Indifference", Discrete Applied Mathematics, 12 , 167-181.

8. McVtie, D. and Wilson, L. (1970). "Stable Marriage Assignment for Unequal Sets." BIT Numerical Mathematics, 10, 295-309.

9. Roth, A. (1984). "The Evolution of the Labor Market for Medical Interns and Residents: A Case Study in Game Theory ", Journal of Political Economy, 92, 991-1016.

10. Roth, A. (1986). "On the Allocation of Residents to Rural Hospitals: A General Property of Two-Sided Matching Markets", Econometrica, $54,425-427$.

11. Roth, A. and Sotomayor, M. (1990). Two-Sided Matching: A Study in Game-Theoretic Modeling and Analysis. Econometric Society Monographs, Vol. 18. Cambridge University Press, Cambridge, England. 
12. Spieker, B. (1985). "The set of super-stable marriages forms a distributive lattice", Discrete Applied Mathematics 58, 79-84.

13. Sotomayor, M. (2011). "The Pareto-stability concept is a natural solution concept for discrete matching markets with indifferences", International Journal of Game Theory, 40, 631-644. 


\section{Appendix}

\section{Strongly stable matchings and the closing property}

Proof of Lemma 1 Let $\mu_{1}, \mu_{2}, \mu_{3} \in \mathcal{M}$ for all $i \in A$.

1. Let's assume that $\mu_{1} I_{i} \mu_{2}$ and $\mu_{2} P_{i} \mu_{3}$. From the definition of $I_{i}$ and $P_{i}$ we have that

$$
\begin{aligned}
& \mu_{1} R_{i} \mu_{2} \text { and } \mu_{2} R_{i} \mu_{1} \\
& \mu_{2} R_{i} \mu_{3} \text { and } \overline{\mu_{3} R_{i} \mu_{2}} .
\end{aligned}
$$

The transitivity of $R_{i}$ and conditions (17), (18) imply that $\mu_{1} R_{i} \mu_{3}$. Suppose that $\mu_{3} R_{i} \mu_{1}$. Condition (17) and transitivity of $R_{i}$ imply that $\mu_{3} R_{i} \mu_{2}$. This contradicts (18). Hence, $\overline{\mu_{3} R_{i} \mu_{1}}$ and $\mu_{1} P_{i} \mu_{3}$.

2. Similarly, we can prove that if $\mu_{1} P_{i} \mu_{2}$ and $\mu_{2} I_{i} \mu_{3}$, then $\mu_{1} P_{i} \mu_{3}$.

3. We assume that $\mu_{1} P_{i} \mu_{2}$ and $\mu_{i}^{\prime} \in\left[\mu_{i}\right]$ for $i=1,2$. Then,

$$
\mu_{1}^{\prime} I_{i} \mu_{1} \text { and } \mu_{1} P_{i} \mu_{2}
$$

Condition (19) and the fact that $\mu_{1} P_{i} \mu_{2}$ imply that

$$
\mu_{1}^{\prime} P_{i} \mu_{2}
$$

We have that $\mu_{1} P_{i} \mu_{2}$ and $\mu_{2} I_{i} \mu_{2}$. By item 2 we have,

$$
\mu_{1}^{\prime} P_{i} \mu_{2}^{\prime}
$$

4. It follows from $\mathbf{1}, \mathbf{2}$ and $\mathbf{3 .}$

Proof of Proposition 1 Let $\left[\mu_{1}\right],\left[\mu_{2}\right],\left[\mu_{3}\right] \in S(R) / \sim$ for all $i \in A$ and let $\mu_{1}^{\prime} \in\left[\mu_{1}\right], \mu_{2}^{\prime} \in\left[\mu_{2}\right]$ and $\mu_{3}^{\prime} \in\left[\mu_{3}\right]$.

1. Reflexivity. Since $R_{M}$ is a reflexive relation over $\mathcal{M}$ we have that

$$
\mu_{1}^{\prime} R_{M} \mu_{1}^{\prime}
$$

so

$$
\left[\mu_{1}\right] R_{M}\left[\mu_{1}\right]
$$

2. Transitivity. Since $R_{M}$ is a transitive relation over $\mathcal{M}$. If $\mu_{1} R_{i} \mu_{2}$ and $\mu_{2} R_{i} \mu_{3}$ then $\mu_{1} R_{i} \mu_{3}$, i.e.,

$$
\left[\mu_{1}\right] R_{M}\left[\mu_{2}\right],\left[\mu_{2}\right] R_{M}\left[\mu_{3}\right] \text { and }\left[\mu_{1}\right] R_{M}\left[\mu_{3}\right]
$$


3. Antisymmetric. Assume that

$$
\left[\mu_{1}\right] R_{M}\left[\mu_{2}\right] \text { and }\left[\mu_{2}\right] R_{M}\left[\mu_{1}\right]
$$

i.e.,

$$
\mu_{1} R_{M} \mu_{2} \text { and } \mu_{2} R_{M} \mu_{1} .
$$

Hence $\mu_{1} I_{M} \mu_{2}$ i.e. $\left[\mu_{1}\right]=\left[\mu_{2}\right]$.

The following Manlove's result will be useful in proof of Proposition 4.

Lemma A1 (Manlove) Let $(M, W, R)$ be a marriage model with indifferences, and let $\mu$ and $\mu^{\prime}$ be two strongly stable matchings. Suppose that for any men $m_{1} \in M, \mu\left(m_{1}\right)=w_{1}$ and $\mu^{\prime}\left(m_{1}\right)=w_{2}$, where $w_{1} \neq w_{2}$, and $w_{2} R_{m_{1}} w_{1}$. Then there are sequences of agents involving, $m_{1}, w_{1}$ and $w_{2}$ as follows: for some $r>1$, there are $r$ men $m_{1}, \ldots . ., m_{r}$ and $r$ women $w_{1}, \ldots, w_{r}$ which satisfies:

$$
\mu\left(m_{i}\right)=w_{i} \quad(1 \leq i \leq r) \text { and } \mu^{\prime}\left(m_{i}\right)=w_{i+1} \quad(1 \leq i \leq r) .
$$

(1) If $w_{2} I_{m_{1}} w_{1}$, then

$$
w_{i+1} I_{m_{i}} w_{i} \quad(1 \leq i \leq r) \text { and } m_{i} I_{w_{i}} m_{i-1} \quad(1 \leq i \leq r) .
$$

(2) If $w_{2} P_{m_{1}} w_{1}$, then

$$
w_{i+1} P_{m_{i}} w_{i} \quad(1 \leq i \leq r) \text { and } m_{i} P_{w_{i}} m_{i-1} \quad(1 \leq i \leq r) .
$$

Where $m_{0}=m_{r}, m_{r+1}=m_{1}$ and $w_{r+1}=w_{1}$.

We could formulate a symmetric Lemma by exchanging the role of women and men.

Proof of Proposition 4 Let $\mu, \mu^{\prime} \in S S(R)$, if $J_{M}\left(\mu, \mu^{\prime}\right)=J_{W}\left(\mu, \mu^{\prime}\right)=\emptyset$, then the result follows. We assume that $J_{M}\left(\mu, \mu^{\prime}\right) \neq \emptyset$. We will show that

$$
J_{M}\left(\mu, \mu^{\prime}\right) \subseteq \mu\left(J_{W}\left(\mu, \mu^{\prime}\right)\right) .
$$

Let $m_{1} \in J_{M}\left(\mu, \mu^{\prime}\right)$. This means that

$$
\mu\left(m_{1}\right) I_{m_{1}} \mu^{\prime}\left(m_{1}\right) .
$$


Since $m_{1} \in J_{M}\left(\mu, \mu^{\prime}\right)$, we have that $\mu\left(m_{1}\right) \neq \mu^{\prime}\left(m_{1}\right)$. Then there exist $w_{1}$ and $w_{2}$ such that $\mu\left(m_{1}\right)=w_{1}$ and $\mu^{\prime}\left(m_{1}\right)=w_{2}$. Since $m_{1} \in J_{M}\left(\mu, \mu^{\prime}\right)$ we have that

$$
w_{1}=\mu\left(m_{1}\right) I_{m_{1}} \mu^{\prime}\left(m_{1}\right)=w_{2} .
$$

By Lemma A1 (Manlove) we have that there exist successions of agents $m_{1}, \ldots m_{r}$ and $w_{1}, \ldots ., w_{r}$ such that

$$
\mu\left(m_{i}\right)=w_{i} \quad(1 \leq i \leq r) \text { and } \mu^{\prime}\left(m_{i}\right)=w_{i+1} \quad(1 \leq i \leq r) .
$$

Also

$$
w_{i+1} I_{m_{i}} w_{i}(1 \leq i \leq r) \text { and } m_{i} I_{w_{i}} m_{i-1}(1 \leq i \leq r) .
$$

If $i=1$ then

$$
m_{1}=\mu\left(w_{1}\right) I_{w_{1}} \mu^{\prime}\left(w_{1}\right)
$$

i.e. $w_{1} \in J_{W}\left(\mu, \mu^{\prime}\right)$. Hence $\mu\left(w_{1}\right)=m_{1} \in \mu\left(J_{W}\left(\mu, \mu^{\prime}\right)\right)$.

Now, we will show that

$$
\mu\left(J_{W}\left(\mu, \mu^{\prime}\right)\right) \subseteq J_{M}\left(\mu, \mu^{\prime}\right) .
$$

Let $\mu\left(w_{1}\right) \in \mu\left(J_{W}\left(\mu, \mu^{\prime}\right)\right)$. Since $w_{1} \in J_{W}\left(\mu, \mu^{\prime}\right)$ we have that

$$
\mu\left(w_{1}\right) I_{w_{1}} \mu^{\prime}\left(w_{1}\right) .
$$

Notice that $\mu\left(m_{1}\right) \neq \mu^{\prime}\left(m_{1}\right)$. Then there exist $m_{1}$ and $m_{2}$ such that $\mu\left(w_{1}\right)=m_{1}$ and $\mu^{\prime}\left(w_{1}\right)=m_{2}$. By Lemma A1 (Manlove) we have that there exist successions of agents $m_{1}, \ldots m_{r}$ and $w_{1}, \ldots ., w_{r}$ such that

$$
\mu\left(w_{i}\right)=m_{i} \quad(1 \leq i \leq r) \text { and } \mu^{\prime}\left(w_{i}\right)=m_{i+1} \quad(1 \leq i \leq r) .
$$

Also

$$
m_{i+1} I_{m_{i}} m_{i} \quad(1 \leq i \leq r) \text { and } w_{i} I_{w_{i}} w_{i-1}(1 \leq i \leq r) .
$$

If $i=1$ then

$$
w_{1}=\mu\left(m_{1}\right) I_{m_{1}} \mu^{\prime}\left(m_{1}\right)
$$

i.e. $m_{1} \in J_{M}\left(\mu, \mu^{\prime}\right)$. Hence $m_{1}=\mu\left(w_{1}\right) \in J_{M}\left(\mu, \mu^{\prime}\right)$.

Proof of Proposition 5 Let $\mu, \mu^{\prime} \in S S S(R)$, if $J_{M}\left(\mu, \mu^{\prime}\right)=J_{W}\left(\mu, \mu^{\prime}\right)=$ $\emptyset$, then the result follows. We assume that $J_{M}\left(\mu, \mu^{\prime}\right) \neq \emptyset$. 
Let $m_{1} \in J_{M}\left(\mu, \mu^{\prime}\right)$. This means that

$$
\mu\left(m_{1}\right) I_{m_{1}} \mu^{\prime}\left(m_{1}\right) .
$$

Since $m_{1} \in J_{M}\left(\mu, \mu^{\prime}\right)$, we have that $\mu\left(m_{1}\right) \neq \mu^{\prime}\left(m_{1}\right)$. Then there exist $w_{1}$ and $w_{2}$ such that $\mu\left(m_{1}\right)=w_{1}$ and $\mu^{\prime}\left(m_{1}\right)=w_{2}$. Since $m_{1} \in J_{M}\left(\mu, \mu^{\prime}\right)$ we have that

$$
w_{1}=\mu\left(m_{1}\right) I_{m_{1}} \mu^{\prime}\left(m_{1}\right)=w_{2} .
$$

Since $\mu, \mu^{\prime} \in S S S(R) \subseteq S S(R)$ we can apply Lemma A1 (Manlove). Then there exist successions of agents $m_{1}, \ldots m_{r}$ and $w_{1}, \ldots ., w_{r}$ such that

$$
\mu\left(m_{i}\right)=w_{i} \quad(1 \leq i \leq r) \text { and } \mu^{\prime}\left(m_{i}\right)=w_{i+1} \quad(1 \leq i \leq r)
$$

Also

$$
w_{i+1} I_{m_{i}} w_{i}(1 \leq i \leq r) \text { and } m_{i} I_{w_{i}} m_{i-1}(1 \leq i \leq r) .
$$

If $i=1$ then

$$
m_{1}=\mu\left(w_{1}\right) I_{w_{1}} \mu^{\prime}\left(w_{1}\right) .
$$

Condition 21 and 22 imply that the pair $\left(m_{1}, w_{1}\right)$ super blocks $\mu^{\prime}$. This contradicts that $\mu^{\prime}$ is a super stable matching. This contradiction came from assuming that $J_{M}\left(\mu, \mu^{\prime}\right) \neq \emptyset$. So the closing property hold.

The next example shows that the closing property is not a necessary condition to the lattice structure of the stable matching set.

Example 6 Let $M=\left\{m_{1}, m_{2}, m_{3}\right\}$ be the set of men and $W=\left\{w_{1}, w_{2}, w_{3}\right\}$ be the set of women. Consider the preference profile $R$ :

$$
\begin{array}{ll}
R_{m_{1}}:\left[w_{1}, w_{2}\right], m_{1} . & R_{w_{1}}: m_{3}, m_{2}, m_{1}, w_{1} . \\
R_{m_{2}}: w_{2}, w_{1}, w_{3}, m_{2} . & R_{w_{2}}: m_{1}, m_{2}, w_{2} . \\
R_{m_{3}}: w_{3}, w_{1}, m_{3} . & R_{w_{3}}: m_{2}, m_{3}, w_{3} .
\end{array}
$$

The set of stable matchings consists of the following matchings:

$\mu_{1}=\left(\begin{array}{ccc}m_{1} & m_{2} & m_{3} \\ w_{1} & w_{2} & w_{3}\end{array}\right), \quad \mu_{2}=\left(\begin{array}{ccc}m_{1} & m_{2} & m_{3} \\ w_{2} & w_{1} & w_{3}\end{array}\right), \quad \mu_{3}=\left(\begin{array}{ccc}m_{1} & m_{2} & m_{3} \\ w_{2} & w_{3} & w_{1}\end{array}\right)$.

Observe that $\mu_{1} R_{m_{i}} \mu_{2} R_{m_{i}} \mu_{3}$, for $i=1,2,3$. Also $\mu_{3} R_{w_{i}} \mu_{2} R_{w_{i}} \mu_{1}$ for $i=1,2,3$. In this example we have a lattice structure over stable matching set. Nevertheless, the stable matching set does not satisfy the closing property. We have

$$
J_{M}\left(\mu_{1}, \mu_{2}\right)=\left\{m_{1}\right\} \text { and } J_{W}\left(\mu_{1}, \mu_{2}\right)=\emptyset .
$$


So

$$
\mu_{1}\left(J_{M}\left(\mu_{1}, \mu_{2}\right)\right)=\mu_{1}\left(m_{1}\right)=w_{1} \neq J_{W}\left(\mu_{1}, \mu_{2}\right) .
$$

